\title{
The Interfacial Layer Effect on Thermal Conductivity of Nano-Colloidal Dispersions
}

\author{
Pasrija R. and Srivastava S.
}

\begin{abstract}
A mathematical model has been proposed for calculating the effective Thermal Conductivity of nano colloidal dispersions by incorporating a thermal conductivity profile for the interfacial layer. The present model has been successfully applied to the colloidal dispersions containing rod shaped nanoparticles as well as those containing spherical nanoparticles. The results have also been compared with other existing models.
\end{abstract}

Index Terms - Thermal conductivity, nanofluids, colloidal dispersion and interfacial layer.

\section{INTRODUCTION}

About a decade ago Choi at Argonne National Laboratory coined a word 'nanofluid' for fluid containing dispersed nanoparticles. Since then nanofluids have attracted a worldwide attention from researchers across the world due to their wide range of potential applications. They are a two phase system with a sold phase and a liquid phase. These find applications in the field of electronics, transportation, space and defense, drug delivery and the areas where energy saving and cooling is the key issue [1]. The major reasons attributed are to their showing intensified heat transfer properties than the conventional solid liquid suspensions are the higher specific surface area, higher stability of dispersion, reduced pumping power and lesser particle clogging. Due to the above stated reasons, some of the thermophysical properties of nanofluids have been found to be enhanced in comparison to the conventionally used base fluids like water, ethylene glycol etc. These include thermal conductivity, thermal diffusivity, viscosity and the heat transfer coefficients. These enhanced properties have been the main driving force for instigating interest amongst the research community towards these nano colloidal dispersions.

There has been a large scale research to study the anomalous enhancement in effective thermal conductivity of nanofluids both theoretically as well as experimentally [2]-[8]. The most commonly employed experimental methods have been Temperature oscillation technique [4], [5] and a Transient hot-wire method [6]-[9] to determine thermal conductivity of the nanofluids. All the experiments have reported unusually higher increase in the effective thermal conductivity values of the nanofluids. About $4 \%$ volume fraction of $\mathrm{Al}_{2} \mathrm{O}_{3}$ nanoparticles when dispersed in water shows an increase of $30 \%$ in thermal conductivity as reported

Manuscript received September 30, 2013; revised January 13, 2014.

R. Pasrija is with DAV College, Abohar (Punjab), India (e-mail: goodluckritu@gmail.com).

S. Srivastava is with Department of Physics, Panjab University, Chandigarh, India (e-mail: Sunita@pu.ac.in). by Masuda et al. [10]. Similarly a 4 volume percent of $\mathrm{CuO}$ nanoparticles with average diameter of $35 \mathrm{~nm}$ in ethylene glycol dispersion show a $20 \%$ increase in thermal conductivity of the dispersion obtained as shown by Eastman et al. [11]. More interestingly, above $100 \%$ enhancement in thermal conductivity in case of CNT based nanofluids. There has been a wide variety of experimental works with a dispersed range of values for different nanoparticle volume fraction which has tempted the research community to look for theoretical physical mechanisms or models that can explain such an anomalous enhancement in effective thermal conductivity of dispersions with nano-sized particles.

A number of theoretical mechanisms proposed so far [12], [13] have been unable to account for one or the other important factors affecting the effective thermal conductivity of a nanofluid These include thermal conductivity of the fluid medium, thermal conductivity of the nanoparticle dispersed, nanoparticle size and its volume fraction, formation of layer around the nanoparticle etc. Yu and Choi [14] have improved the Maxwell model to incorporate the concept of interfacial layer as a more ordered layer is found to have major impact on the nanofluids thermal conductivity. But this model remained silent about the interaction between nanoparticles and the fluid medium. Many interfacial layer models have come up so far which presented empirical formulae for describing the profile of thermal conductivity of the Interfacial nanolayer. Some of these include Tillman et al. [15], Nsofor et al. [16] and Xie et al. [17] among others.

In the present theoretical formulation, a general empirical form of thermal conductivity profile inside the interfacial layer has been proposed. This is then used to develop a model for calculating the effective thermal conductivity of the colloidal dispersion containing nanoparticles. The parameters involved in the present study are the effect of Nanoparticle size, the volume fraction of the nanoparticles, thermal conductivity of the base fluid and the thermal conductivity of the nanoparticle itself.

\section{THEORETICAL FORMULATION}

\section{A. The Nanoparticle Structure and the Thermal Conductivity Profile}

The equivalent nanoparticle consists of nanoparticle and interfacial nanolayer consisting of the orderly arrangement of base fluid molecules around the nanoparticles. This nanoparticle is dispersed in the base fluid forming a colloidal suspension called 'nanofluid' or nano colloidal dispersion. This orderly arrangement is expected to have intermediate thermal conductivity profile between the base fluid and the dispersant nanoparticle. The nanoparticle structure is 
depicted in the Fig. 1.

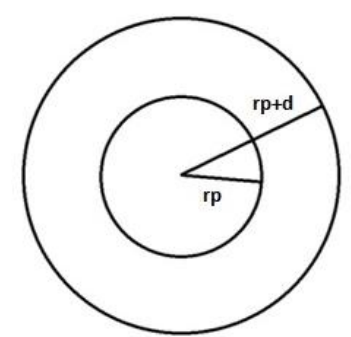

Fig. 1. The nanoparticle structure.

The thermal conductivity of the nanolayer is supposed to vary with distance $\mathrm{r}$ from inner radius $r_{p}$ of the nanoparticle to the entire thickness of the interfacial layer around the nanoparticle before it becomes equal to the $k_{f}$ at the interface. Here, the empirical formulation is introduced based on the assumptions that thermal conductivity equals the thermal conductivity of the nanoparticle $k_{p}$ at the inner interface and that at the outer interface it is equal to base fluid's thermal conductivity i.e $k_{f}$ so that

$$
k(r)=\left\{\begin{array}{lc}
k_{p} & \text { size } \leq r_{p} \\
f(r) & r_{p} \leq r \leq r_{p}+d \\
k_{f} & r_{p}+d \leq \text { size }
\end{array}\right.
$$

Size in the equation (1) refers to radius of the nanoparticle Here, the following tangential variation of $f(r)$ is proposed

$$
f(r)=k_{p}+\frac{\left(k_{f}-k_{p}\right)}{\tanh m} \tanh \left(m \frac{\left(r-r_{p}\right)}{d}\right)
$$

where, $m$ is a real positive integer. The corresponding thermal conductivity profile within the interfacial layer is displayed in Fig. 2.

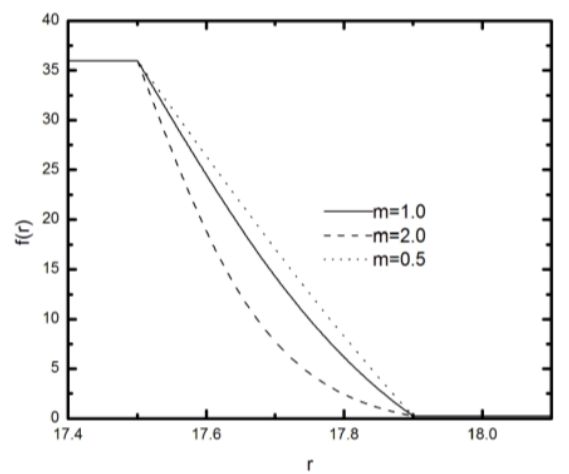

Fig. 2. The thermal conductivity profile versus radius of the nanoparticle.

The above graph shows the thermal conductivity profile of the Interfacial nanolayer for $\mathrm{Al}_{2} \mathrm{O}_{3}$ nanoparticles in Ethylene Glycol with size of $35 \mathrm{~nm}$. The graph is plotted for different values of $\mathrm{m}(m=1,2,0.5)$. The value of $\mathrm{m}$ as decreased from 1.0 shift towards a linear profile while $\mathrm{m}$ if increased from 1.0 show a fast decrease moving towards being a step function profile. In the present model the value of $m$ is taken as 1 in each type of nanofluid system.

\section{B. Dispersions with Spherical Nanoparticles}

It is assumed that the $\mathrm{Al}_{2} \mathrm{O}_{3}$ and $\mathrm{CuO}$ nanoparticles are spherical when dispersed in base fluids, ethylene Glycol and water respectively. The thermal conductivity of the interfacial layer is a continuous function of $r$ within the nanolayer. The thermal resistance offered by the nanolayer is given by

$$
R_{L}=\frac{1}{4 \pi k}\left(\frac{1}{r_{p}}-\frac{1}{r_{p}+d}\right)
$$

Also the resistance is related to the thermal conductivity distribution between $r_{p}$ and $r_{p}+d$ as

$$
R_{L}=\int_{r p}^{r p+d} \frac{d r}{4 \pi r^{2} f(r)}
$$

Combining the preceding two equations, we get

$$
k_{l}=\frac{d}{r_{p}\left(r_{p}+d\right) \int_{r_{p}}^{r_{p}+d} \frac{d r}{r^{2} f(r)}}
$$

\section{Dispersions with Rod Shaped Nanoparticle}

In case the nanoparticles are assumed to be cylindrical like rod shaped nanoparticles then the thermal resistance offered by the nanolayer will be given by

$$
R_{L}=\frac{1}{\pi K_{l}}\left[\frac{l}{r_{p}^{2}}-\frac{(l+2 d)}{\left(r_{p}+d\right)^{2}}\right]
$$

where $l X r_{p}$ are the dimensions of the nanoparticle

And the resistance is related to the thermal conductivity distribution between $r_{p}$ and $r_{p}+d$ as

$$
R_{L}=\int_{r_{p}}^{r_{p}+d} \frac{d r}{2 \pi r_{p}\left(r_{p}+l\right) f(r)}
$$

Using the two preceding equations we get

$$
k_{l}=\frac{\left[\frac{l}{r_{p}^{2}}-\frac{(l+2 d)}{\left(r_{p}+d\right)^{2}}\right]}{\int_{r_{p}}^{r_{p}+d} \frac{d r}{2 r_{p}\left(r_{p}+l\right) f(r)}}
$$

D. The Expression for the Effective Thermal Conductivity

The effective thermal conductivity is defined using Fourier's law of heat conduction as

$$
\langle\vec{q}\rangle=-k_{e f f}\langle\nabla T\rangle
$$


Here $\langle\vec{q}\rangle$ is the average heat flux and $\nabla T$ is the temperature gradient vector. The mixture is assumed to be statistically homogeneous and isotropic. The effective thermal conductivity $\mathrm{k}_{\mathrm{eff}}$ of the nanoparticle containing dispersion can be obtained by combining the contributions from the fluid, the nanoparticles and the nanolayer as

$$
<\vec{q}>=-k_{f}<\nabla T>+<\vec{q}_{p}>+<\vec{q}_{l}>
$$

Lu and Song [18] used equilibrium hard sphere fluid model and determined the effective thermal conductivity by considering the inclusion which is the dispersed nanoparticle to be coated and debonded for two interacting particles. They considered temperature field to be linear and applied the ambient temperature gradient as unity along a specified direction to the system. The effective thermal conductivity is computed to be given by

$$
k_{\text {eff }}=k_{f}\left[1+3 F \phi_{T}+\frac{3 F^{2} \phi_{T}^{2}}{1-F \phi_{T}}\right]
$$

with

$$
F=\frac{\alpha_{1}\left[(1+\beta)^{3}-\frac{\alpha_{2}}{\alpha_{3}}\right]}{(1+\beta)^{3}+2\left(\alpha_{1}\right)\left(\alpha_{2}\right)}
$$

and

$$
\alpha_{1}=\frac{k_{p}-k_{l}}{k_{p}+2 k_{l}}, \alpha_{2}=\frac{k_{l}-k_{f}}{k_{l}+2 k_{f}}, \alpha_{3}=\frac{k_{f}-k_{l}}{k_{f}+2 k_{l}}
$$

The total volume fraction of the equivalent nanoparticle which is formed from the original nanoparticle of radius $r_{p}$ and the nanolayer of thickness $d$ is given by

$$
\phi_{T}=\frac{4}{3} \pi\left(r_{p}+d\right)^{3} n=\phi(1+\beta)^{3}
$$

where

$$
\phi=\frac{4}{3} \pi r_{p}^{3} n
$$

is the original volume fraction of the nanoparticle without including the nanolayer and $\mathrm{n}$ defines the number density of particles and $\beta=d / r_{p}$.

\section{GRAPHS AND DISCISSION}

Equation (11) is used to study the effective thermal conductivity ratio $\left(k_{e f f} / k_{f}\right)$ of the nanoparticle dispersion and its relationship with the volume fraction and the effect of size on effective thermal conductivity is also studied using the same expression for the nanofluid. Fig. 3 below shows the variation of thermal conductivity ratio at different interfacial layer thickness with the size of the nanoparticle. The ratio decreases abruptly with the increase in size of the nanoparticle as well as with the decrease in interfacial layer thickness. The graph indicates that the nanofluid system shows higher thermal conductivity at smaller radius of the nanoparticle and higher interfacial layer thickness. The systems studied for spherical shaped nanoparticle dispersions are Alumina in ethylene glycol and copper oxide in water taking the size of nanoparticles to be $35 \mathrm{~nm}$ and $23.6 \mathrm{~nm}$ respectively.

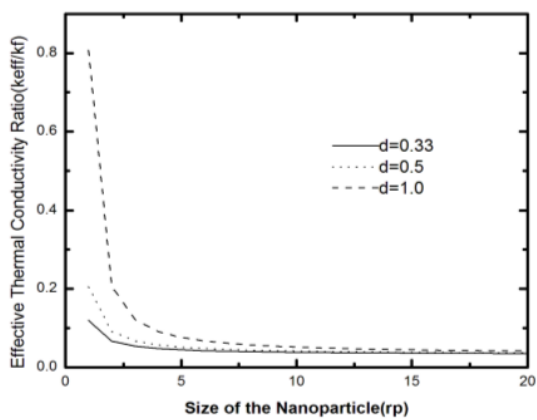

Fig. 3. The effective thermal conductivity ratio versus size of the nanoparticle.

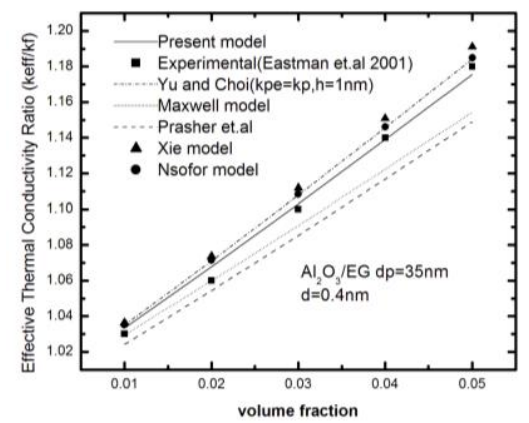

Fig. 4. The variation of effective thermal conductivity ratio with volume fraction for $\mathrm{Al}_{2} \mathrm{O}_{3} / \mathrm{EG}$ nano-dispersion.

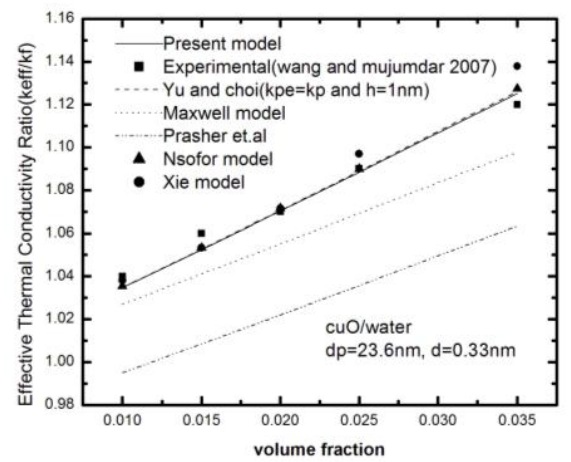

Fig. 5. The variation of effective thermal conductivity ratio with volume fraction for $\mathrm{CuO} /$ Water nano-dispersion.

The above graphs show that the effective thermal conductivity ratio increases with an increase in the volume fraction of the nano-dispersants in the base fluid. The results from this model have been compared with other theoretical models like Yu and Choi [14], Maxwell model [12] and Prasher model [19], [20].

The maximum deviation in case of $\mathrm{Al}_{2} \mathrm{O}_{3} / \mathrm{EG}$ nanodispersion is $0.75 \%$ in the present model whereas it is $1.3 \%$ in case of Xie model [17] and it is $1.07 \%$ in case of Nsofor model [16]. The maximum deviation in case of 
$\mathrm{CuO} /$ Water nanodispersion is $0.7 \%$ in the present model and the maximum deviation in case of Xie model is $1.6 \%$ and in case of Nsofor model is $0.6 \%$. In case of TiO2/EG our maximum deviation from experimental data is $0.5 \%$. Also in case of $\mathrm{CuO} /$ water system, the present model overlaps the model by $\mathrm{Yu}$ and Choi when the thermal conductivity of the equivalent particle is taken to be equal to the thermal conductivity of the nanoparticle with an interfacial layer thickness of $1 \mathrm{~nm}$. The value for the interfacial layer thickness in each type of nanofluid system has been calculated using least square fitting method.

The system studied for rod shaped nanoparticle dispersion is $\mathrm{TiO}_{2} / \mathrm{EG}$ and the available experimental data by Murshed et al. [9] is used for comparison.

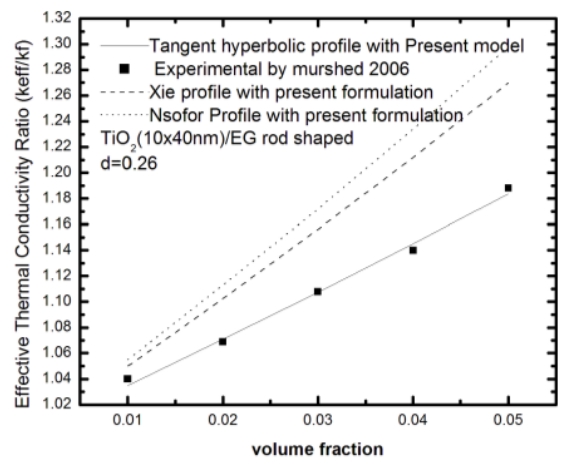

Fig. 6. The variation of effective thermal conductivity ratio with volume fraction for $\mathrm{TiO}_{2} / \mathrm{EG}$ system.

It is evident from the Fig. 6 that the proposed thermal conductivity profile (tangent hyperbolic) fits well with the avaliable experimental values by Murshed [9]. It shows the comparison with the thermal conductivity profiles given by Xie et al. ( linear Profile) and Nsofor et al. (Quadratic Profile).

\section{CONCLUSION}

The present model successfully explains the anomalous behavior of effective thermal conductivity of nanofluids containing spherical as well as cylindrical nanoparticles. The maximum deviation from the experimental result is $0.75 \%$ in case of present proposed semi empirical model.

\section{ACKNOWLEDGMENT}

The authors are highly obliged to Dr. Vishwamittar for his useful comments and suggestions.

\section{REFERENCES}

[1] K. V. Wong and O. De Leon, "Applications of Nanofluids: Current and Future," Advances in Mechanical Engineering, pp. 1-11, 2010.

[2] S. U. S. Choi, Z. G. Zhang, W. Yu, F. E. Lockwood, and E. A. Grulke, "Anomalous thermal conductivity enhancement in nanotube suspensions," Appl. Phys. Lett., vol. 79, pp. 2252-2254, August 2001.

[3] X. Wang, X. Xu, and S. U. S. Choi, "Thermal conductivity of nanoparticle-fluid mixture," Journal of Thermophysics and Heat Transfer, vol. 13, no. 4, pp. 474-480, October-December 1999.

[4] S. K. Das, N. Putra, P. Thiesen, and W. Roetzel, "Temperature dependence of thermal conductivity enhancement for nanofluids," ASME J. Heat Trans., vol. 125, pp. 567-74, July 2003.

[5] W. Roetzel, S. Prinzen, and Y. Xuan, "Measurement of Thermal Diffusivity Using Temperature Oscillations," in Thermal Conductivity 21, C. Y. Cremers and H. A. Fine, Eds., New York, Plenum Press, 1990, pp. 201-207.
[6] W. Czarnetzki and W. Roetzel, "Temperature Oscillation techniques for simultaneous measurement of thermal diffusivity and conductivity," International Journal of Thermophysics, vol. 16, pp. 413-422, March 1995

[7] T. P. Teng, Y. H. Hung, T. C. Teng, H. E. Mo, and H. G. Hsu, "The effect of alumina/water nanofluid particle size on thermal conductivity," Appl. Thermal Engineering, vol. 30, pp. 2213-2218, October 2010.

[8] W. Duangthongsuk and S. Wongwises, "Measurement of temperature-dependent thermal conductivity of $\mathrm{TiO}_{2}$-water nanofluids," Experimental thermal and Fluid Science, vol. 33, pp. 706-714, April 2009.

[9] S. M. S. Murshed, K. C. Leong, and C. Yang, "Investigations of thermal conductivity and viscosity of nanofluids," International Journal of Thermal Sciences, vol. 47, pp. 560-568, June 2008.

[10] H. Masuda, A. Ebata, K. Teramae, and N. Hishinuma, " Alternation of Thermal Conductivity and viscosity of liquid by Dispersing Ulltra-fine particles (Dispersion of $\gamma-\mathrm{Al}_{2} \mathrm{O}_{3}, \mathrm{SiO}_{2}$ and $\mathrm{TiO}_{2}$ Ultra-fine particles," Netsu Bussei (Japan), vol. 4, pp. 227-233, 1993.

[11] J. A. Eastman, S. U. S. Choi, S. Li, W. Yu, and L. J. Thompson, "Anomalously increased thermal conductivity of ethylene based nanofluids containing copper nanoparticles," Appl. Phys. Lett., vol. 78 pp. 718-720, February 2001.

[12] J. C. Maxwell, A Treatise on Electricity and Magnetism, $2^{\text {nd }}$ ed., Clarendon Press, U.K.: Oxford, Vol. 1, 1881, pp. 435-441.

[13] R. L. Hamilton and O. K. Crosser, "Thermal conductivity of heterogeneous two-component systems," IEC Fundam., vol. 1, no. 3, pp. 187-191, August 1962.

[14] W. Yu and S. U. S. Choi, "The role of interfacial layers in the enhanced thermal conductivity of nanofluids: a renovated Maxwell model," $J$. Nanoparticle Res., vol. 5, pp. 167-171, April 2003.

[15] P. Tillman and J. M. Hill, "Determination of nanolayer thickness for a nanofluid," International Communications in Heat and Mass Transfer vol. 34, pp. 399-407, February 2007.

[16] E. C. Nsofor and T. Gadge, "Investigations on the nanolayer heat transfer in nanoparticles-in-liquid suspensions," ARPN Jl. Of Engg \& Appl.Sc., vol. 6, pp. 21-28, January 2011.

[17] H. Xie, M. Fujii, and X. Zhang, "Effect of interfacial layer on the effective thermal conductivity of nanoparticle-fluid mixture," Int. $J$. Heat and Mass Transfer, vol. 48, pp. 2926-2932, October 2005.

[18] S. Y. Lu and J. L. Song, "Effective thermal conductivity of composites with spherical inclusions: effect of coating and detachment," J. Appl. Phys., vol. 79, pp. 609-618, January 1996.

[19] R. Prasher, P. Bhattacharya, and P. E. Phelan, "Brownian motion based convective-conductive model for the effective thermal conductivity of nanofluids," ASME J. Heat Transfer, vol. 128, pp. 588-595, November 2006.

[20] X. Q. Wang and A. S. Mujumdar, "Heat transfer characteristics of nanofluids: A Review," Int. J. Thermal Sci, vol. 46, pp. 1-19, June 2007.

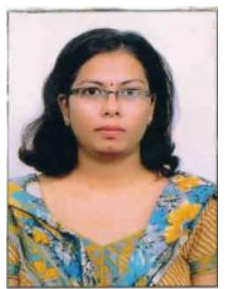

R. Pasrija has done her masters in science from Panjab University Chandigarh, India in 2003. She is presently teaching as an assistant professor at P.G. Department of Physics, DAV College, Abohar, Punjab, India.

She is presently working for her $\mathrm{PhD}$ from Department of Physics, Panjab University Chandigarh. She is a life member of Indian Assosiation of Physics Teachers.

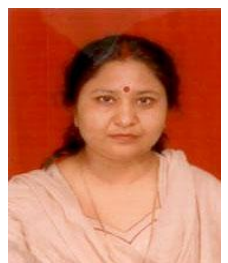

S. Srivastava has obtained her doctor's degree from Panjab University, Chandigarh in 1992 in the field of general theoretical Physics particularly on non linear Oscillators. She completed her M.Phil in 1987 and Masters in Science in 1985 from Panjab University. Her major field of study is Condensed Matter Theory. She is at present an assistant professor at Department of Physics at Panjab University, Chandigarh. She has about 50 research papers to her credit with 36 papers in Journals of International repute. She has authored a chapter "Restricted flow in Nano-Channels" in a book "Nanotechnology Research Progress", edited by Julian F. Vogel and Felix T. Jung (Nova Publishers, New York, 2009). Her present research interest includes confinement of fluids in nanogeometries and nanofluids

Dr. Srivastava is a life member of Indian Association of Physics Teachers and Indian Physics Association. 